\title{
Characteristics of foodborne outbreaks in which use of analytical epidemiological studies contributed to identification of suspected vehicles, European Union, 2007 to 2011
}

\author{
K. M. SCHLINKMANN ${ }^{1,2,3}$, O. RAZUM ${ }^{2}$ AND D. WERBER ${ }^{3,4 *}$ \\ ${ }^{1}$ Helmholtz Centre for Infection Research, Department for Epidemiology, ESME-Epidemiological and \\ Statistical Methods Research Group \\ ${ }^{2}$ Bielefeld University, School of Public Health, Department of Epidemiology and International Public Health \\ ${ }^{3}$ Robert Koch-Institute, Department of Infectious Disease Epidemiology, Unit for Gastrointestinal Infections, \\ Zoonoses and Tropical Infections \\ ${ }^{4}$ State Health Office for Health and Social Affairs, Berlin, Germany
}

Received 8 June 2016; Final revision 14 December 2016; Accepted 18 December 2016; first published online 6 February 2017

\section{SUMMARY}

Foodborne disease outbreaks (FBDOs) occur frequently in Europe. Employing analytical epidemiological study designs increases the likelihood of identifying the suspected vehicle(s), but these studies are rarely applied in FBDO investigations. We used multivariable binary logistic regression analysis to identify characteristics of investigated FBDOs reported to the European Food Safety Authority (2007-2011) that were associated with analytical epidemiological evidence (compared to evidence from microbiological investigations/descriptive epidemiology only). The analysis was restricted to FBDO investigations, where the evidence for the suspected vehicle was considered 'strong', i.e. convincing. The presence of analytical epidemiological evidence was reported in $2012(50 \%)$ of these 4038 outbreaks. In multivariable analysis, increasing outbreak size, number of hospitalizations, causative (i.e. aetiological) agent (whether identified and, if so, which one), and the setting in which these outbreaks occurred (e.g. geographically dispersed outbreaks) were independently associated with presence of analytical evidence. The number of investigations with reported analytical epidemiological evidence was unexpectedly high, likely indicating the need for quality assurance within the European Union foodborne outbreak reporting system, and warranting cautious interpretation of our findings. This first analysis of evidence implicating a food vehicle in FBDOs may help to inform public health authorities on when to use analytical epidemiological study designs.

Key words: Communicable diseases, disease outbreaks, epidemiological studies, foodborne diseases, public health surveillance.

\section{INTRODUCTION}

Foodborne diseases are an important and continuing public health problem comprising a broad variety of

\footnotetext{
* Author for correspondence: PD Dr. med. vet. D. Werber, State Office for Health and Social Affairs, Unit for infectious disease surveillance and environmental health, Darwinstraße 15, 10589 Berlin, Germany.

(Email: Dirk.Werber@lageso.berlin.de)
}

illnesses [1-3]. Everyone is basically susceptible for these diseases, but young children, the elderly, pregnant women, and immunocompromised persons are considered to be particularly vulnerable [4]. With the ageing of populations in the European Union (EU), an increase in morbidity and mortality from foodborne diseases is expected [5]. Most foodborne diseases have a microbial aetiology and occur sporadically, i.e. without an apparent epidemiological 
link to other cases. According to the zoonosis directive [6], a foodborne disease outbreak (FBDO) is defined as 'an incidence, observed under given circumstances, of two or more human cases of the same disease and/or infection, or a situation in which the observed number of human cases exceeds the expected number and where the cases are linked, or are probably linked, to the same food source' [6, p. 33]. The causative (i.e. aetiological) agents in these outbreaks are mostly microbial agents or microbial toxins [2]. FBDOs are common and responsible for a high burden of disease in populations and also lead to deaths in developing as well as in developed countries [1,7]. In Europe, a total of 5363 FBDOs were recorded in 2012 [8, 9], affecting 55453 people of whom 5118 were hospitalized and 41 died [9].

FBDOs need to be systematically investigated to understand their epidemiology. Successful outbreak investigations provide important insights into the causative agent, the suspected food vehicle, as well as factors in food preparation or handling contributing to the outbreak $[10,11]$. The chance of identifying a suspected vehicle can be increased through the application of analytical epidemiological studies (principally cohort studies and case-control studies) [10, 12]. However, these studies are not commonly applied in outbreak investigations [8, 10], particularly not by local health departments, which usually represent the competent authority in local FBDOs, and most FBDOs are local [8].

Foodborne outbreaks in the EU have to be investigated by the competent authorities of EU member states [6]. Member states have to report FBDOs to European Food Safety Authority (EFSA), which, in essence, constitutes the EU foodborne outbreak reporting system. EFSA lays down a set of technical specifications of how the results of investigations of FBDOs have to be reported. The results of these investigations are categorized according to the strength of evidence provided for a specific food vehicle. 'Strong' evidence requires convincing results provided by the epidemiological investigation (descriptive or through analytical study designs), microbiological investigation, or both [11].

The aim of this study was to identify characteristics of investigated FBDOs, reported to EFSA, in which analytical epidemiological studies contributed to strong evidence for the suspected vehicle(s), compared to non-analytical evidence (i.e. microbiological or descriptive epidemiological methods only).

\section{MATERIALS AND METHODS}

\section{Data source}

A dataset on FBDOs in Europe was provided by EFSA for analysis. It consisted of data of FBDOs from the member states for the years 2007-2011, in which the strength of the evidence for the suspected vehicle was considered strong as only those have to be reported in detail [11].

\section{Variables}

The nature of evidence for the suspected food vehicle, i.e. epidemiological, microbiological, or both, was reported. The outcome of interest was 'analytical epidemiological evidence', defined as 'a statistically significant association between consumption of a food vehicle and being a case in an outbreak demonstrated by study designs such as a cohort study, a case-control study or similar studies' [11, p. 5]. The outcome variable 'analytical evidence' was created with a binary outcome ('yes'-presence of analytical epidemiological evidence; 'no' - microbiological or descriptive epidemiological evidence only). Thus, the comparison group were FBDOs in which strong evidence for the suspected vehicle was provided by microbiological evidence, descriptive epidemiological evidence (since 2010), or both (since 2010).

We included the following eight reported characteristics of a FBDO as potential explanatory variables in the analysis (definitions based on EFSA [11]): (i) causative agent (bacterium, virus, parasite, or toxin that was detected and/or isolated in the course of the investigation and which is 'considered to be the cause of the FBDO'), (ii) deaths (reported number of outbreak cases who died as a result of the FBDO), (iii) hospitalizations (known number of outbreak cases in the FBDO who were hospitalized), (iv) outbreak size (number of all persons meeting the outbreak case definition, including those who were hospitalized or who died as a result of the FBDO), (v) setting (location where the food was consumed), (vi) place of origin (place, other than setting, where the contamination or the mishandling of the implicated food occurred), (vii) severity of outbreak (severe if at least one person died as a result of the outbreak or at least ten persons or $25 \%$ were hospitalized; self-constructed variable), (viii) type of outbreak (general, i.e. affecting persons living in more than one household, vs. single household).

The categories of the variable setting were collapsed, where deemed plausible, for ease of analysis 
Table 1. Frequency of outbreak characteristics of foodborne disease outbreaks (FBDOs) reported to European Food Safety Authority (EFSA) 2007-2011, in which the evidence for the suspected vehicle was 'strong'*

\begin{tabular}{|c|c|c|c|c|}
\hline & \multirow[b]{2}{*}{ Analytical evidence, $N$} & \multirow[b]{2}{*}{ No analytical evidence, $N$} & \multicolumn{2}{|l|}{ Total } \\
\hline & & & $N$ & $\%$ \\
\hline \multicolumn{5}{|l|}{ Causative agent } \\
\hline Bacillus spp. & 111 & 144 & 255 & $6 \cdot 3$ \\
\hline Calicivirus & 119 & 188 & 307 & $7 \cdot 6$ \\
\hline Campylobacter spp. & 63 & 79 & 142 & $3 \cdot 5$ \\
\hline Clostridium $\dagger$ & 98 & 120 & 218 & $5 \cdot 4$ \\
\hline Histamine & 81 & 98 & 179 & $4 \cdot 4$ \\
\hline Listeria monocytogenes & 2 & 11 & 13 & $0 \cdot 3$ \\
\hline Marine biotoxin & 19 & 17 & 36 & $0 \cdot 9$ \\
\hline Mushroom & 2 & 77 & 79 & $2 \cdot 0$ \\
\hline Other & 45 & 73 & 118 & $2 \cdot 9$ \\
\hline Salmonella spp. & 642 & 853 & 1495 & $37 \cdot 0$ \\
\hline Shigella spp. & 23 & 13 & 36 & $0 \cdot 9$ \\
\hline Staphylococcus & 240 & 180 & 420 & $10 \cdot 4$ \\
\hline Trichinella & 6 & 113 & 119 & $2 \cdot 9$ \\
\hline Unknown & 561 & 60 & 621 & $15 \cdot 4$ \\
\hline \multicolumn{5}{|l|}{ Hospitalizations } \\
\hline 0 case & 1027 & 755 & 1782 & $44 \cdot 1$ \\
\hline 1 case & 248 & 237 & 485 & $12 \cdot 0$ \\
\hline $2-3$ cases & 333 & 433 & 766 & $19 \cdot 0$ \\
\hline$\geqslant 4$ & 226 & 418 & 644 & $15 \cdot 9$ \\
\hline Missing & 178 & 183 & 361 & $8 \cdot 9$ \\
\hline \multicolumn{5}{|l|}{ Outbreak size } \\
\hline $2-3$ cases & 599 & 564 & 1163 & $28 \cdot 8$ \\
\hline 4-7 cases & 443 & 513 & 956 & $23 \cdot 7$ \\
\hline $8-18$ cases & 444 & 477 & 921 & $22 \cdot 8$ \\
\hline$\geqslant 19$ & 514 & 450 & 964 & $23 \cdot 9$ \\
\hline Missing & 12 & 22 & 34 & $0 \cdot 8$ \\
\hline \multicolumn{5}{|l|}{ Setting } \\
\hline Commercial food establishment & 573 & 589 & 1162 & $28 \cdot 8$ \\
\hline Disseminated cases§ & 12 & 20 & 32 & $0 \cdot 8$ \\
\hline Household\| & 734 & 920 & 1654 & $41 \cdot 0$ \\
\hline Institution & 474 & 295 & 769 & $19 \cdot 0$ \\
\hline Other\# & 186 & 133 & 319 & $7 \cdot 9$ \\
\hline Unknown & 33 & 68 & 101 & $2 \cdot 5$ \\
\hline Missing & 0 & 1 & 1 & $0 \cdot 0$ \\
\hline
\end{tabular}

* Univariable significant variables (analysis adjusted for reporting country and year of notification).

$\dagger$ Clostridium perfringens and Clostridium botulinum.

$\$$ Restaurant/café/pub/bar/hotel, mobile retailer/market/street vendor, takeaway or fast-food outlet, temporary mass catering (fairs, festivals).

$\S$ Geographically dispersed outbreaks.

\| Household, domestic kitchen, camp, picnic.

T Canteen or workplace catering, hospital/medical care facilities, residential institution (nursing home, prison, boarding schools), school, kindergarten.

\# Other, farm (primary production) $(n<10)$, aircraft/ship/train $(n<10)$.

and interpretation. All categories with $n<10$ were combined into one category. The continuous variables outbreak size and hospitalization were categorized on the basis of quartiles (Table 1).

The following three variables concerning the suspected food vehicle were excluded from analysis because they were a result (an effect) of the FBDO investigation and thus could not influence the preceding kind of FBDO investigation and the nature of the evidence (definitions based on EFSA [11]) provided (reverse causation): (i) vehicle (food considered to have been the vehicle of the causative agent or its 
toxins, reported in categories), (ii) vehicle information (detailed information on kind of food) and (iii) vehicle origin (food vehicle originated from domestic market, from intra-EU trade, or was imported from outside EU).

\section{Statistical analysis}

In univariable analyses, the relationship between the presence of analytical epidemiological evidence and the eight outbreak characteristics was evaluated using binary regression analysis. We used the likelihood ratio test to assess statistical significance. Variables with a $P$ value $<0.05$ were considered candidate variables for multivariable analysis. Multicollinearity was investigated by determining the variance inflation factor with a threshold value of $\leqslant 3$.

Characteristics associated with analytical evidence in univariable analysis were further assessed using a multivariable binary logistic regression model. Candidate variables were offered to the model using a forward stepwise selection approach with a threshold value of $P<0 \cdot 05$. Goodness of the model fit was evaluated by computing the pseudo-coefficient of determination, i.e. Nagelkerke's $R^{2}$. Year of reporting and reporting country were forced into the model for adjustment. These adjustments were required for two reasons: first, in 2009 a change in the technical reporting specifications occurred. From 2007 to 2009, according to EFSA's technical specifications, strong evidence could only be provided by FBDOs in which the suspected vehicle was identified by use of an analytical epidemiological study or in which the causative agent was detected in the suspected food vehicle. From 2010 onwards, strength of evidence, judged by the investigators, was based on all available evidence allowing for more lines of evidence to contribute to strong evidence for a suspected food vehicle than before [11]. Second, the frequency of investigations of FBDOs with strong evidence varied widely with a few countries accounting for most investigations of FBDOs with strong evidence from analytical study designs.

\section{RESULTS}

\section{Descriptive results}

The EFSA dataset consisted of 4102 foodborne outbreaks with strong evidence for a suspected food vehicle. We excluded aggregated information on more than one outbreak $(n=38)$ and outbreaks where $<2$ cases were involved $(n=26)$. This reduced the dataset to 4038 FBDOs. The presence of analytical epidemiological evidence was reported in 2012 $(50 \%)$ of these 4038 outbreaks.

The comparison group consisted of FBDOs in which microbiological evidence was reported as the only line of evidence $(73 \%)$ or in combination with descriptive epidemiological evidence $(6 \%)$ or in which only descriptive epidemiological evidence was present $(19 \%)$; in $2 \%$ the nature of evidence was not reported. The proportion of investigations of FBDOs with analytical epidemiological evidence for the suspected vehicle decreased from 2010 onwards (Fig. 1).

The median number of affected persons in FBDOs, reported to EFSA from 2007 to 2011, was 7 [interquartile range (IQR) 3-18, min-max 2-20 000]. In around half $(47 \%)$ of the FBDOs, at least one person was reported to be hospitalized per outbreak (median 1, IQR 0-3, min-max 0-11 352); 143 deaths were reported in $49(1 \%)$ outbreaks. Salmonella spp. was the most frequently reported causative agent (37\%). Most reported FBDOs occurred in households (41\%), followed by those in commercial food establishments $(29 \%)$. FBDOs in institutions were often (62\% of all institutional outbreaks) associated with analytical evidence (Table 1).

\section{Multivariable results}

Four variables were selected for multivariable analysis: causative agent, hospitalization, outbreak size, and setting. All remained in the final model with a Nagelkerke's $R^{2}$ of $0 \cdot 472$. In total 3665 outbreaks were considered in the multivariable analysis (Table 2).

Causative agent. Most notably, the odds of being an outbreak, where the causative agent was not reported (vs. outbreaks caused by Salmonella spp.), was 15 times higher in investigations with analytical evidence than without. Further, causative agents associated with analytical epidemiological evidence were Bacillus spp., calicivirus, Campylobacter spp., histamine, Shigella spp., and Staphylococcus.

Hospitalization. The number of hospitalized persons was negatively associated with FBDO investigations with analytical epidemiological evidence.

Outbreak size. There was a positive association between an outbreak with analytical epidemiological evidence and the outbreak size. 


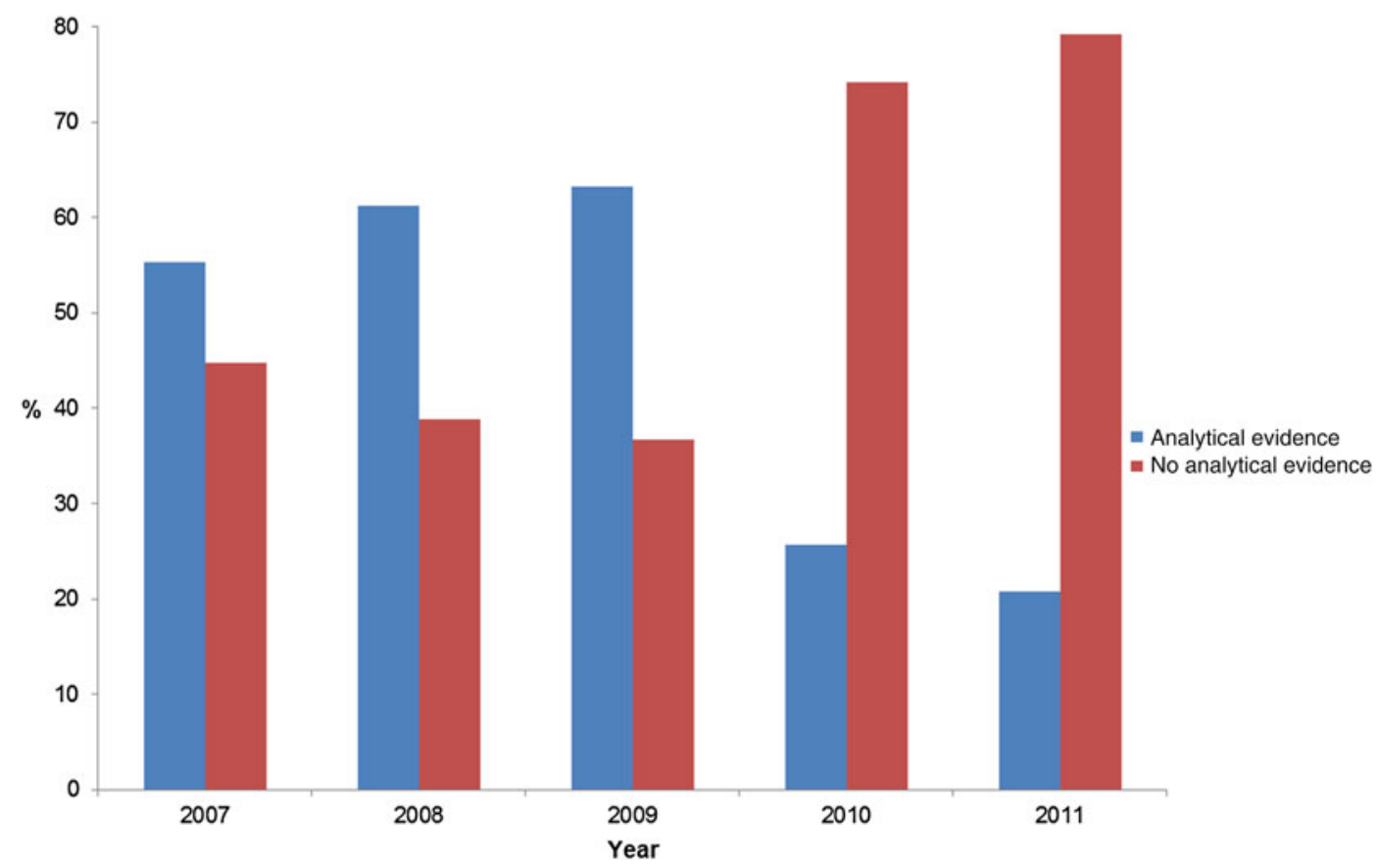

Fig. 1. Proportion of foodborne disease outbreaks (FBDOs) with 'strong' evidence for a food vehicle reported to European Food Safety Authority (EFSA) from 2007-2011, which reported use of an analytical epidemiological study, by reporting year. Number of FBDOs by reporting year: 2007 (1556), 2008 (690), 2009 (766), 2010 (493), 2011 (533).

Setting. Most notably, the odds of being an outbreak with disseminated (i.e. geographically dispersed) cases ( $v s$. household cases) was almost seven times higher in outbreaks with analytical evidence than the odds of being an outbreak with disseminated cases ( $v s$. household cases) in FBDOs with microbiological/descriptive epidemiological evidence only. Furthermore, an association with investigations with analytical epidemiological evidence was noted for FBDOs in institutional and commercial settings (Table 2).

\section{DISCUSSION}

Timely investigation with the aim of controlling FBDOs is an important public health task [13]. Targeted interventions rely on identifying the contaminated food, i.e. on investigations that provide strong (convincing) evidence for a food vehicle. We analysed data on 4038 such investigations reported in Europe from 2007 to 2011 to better understand the characteristics of FBDOs, where use of analytical study designs (vs. microbiological investigations/ descriptive epidemiology only) contributed to the strong evidence implicating a particular food vehicle. Characteristics associated with the presence of analytical epidemiological evidence in investigations of
FBDOs were the causative agent, number of hospitalized cases, outbreak size, and setting. We identified statistical associations of analytical epidemiological study designs with geographically diffuse FBDOs, FBDOs occurring in institutional settings, and with FBDOs in which a pathogen had not (yet) been identified. Furthermore, analytical epidemiological evidence was associated with an increasing number of outbreak cases.

Our results are plausible and may provide an evidence base for recommendations for their successful use. For example, in institutional settings the list of potential suspects is confined to food vehicles served by the institution, allowing for targeted and timely exposure assessment and hypothesis generation. In addition, institutions usually keep records of their food purchases and food from the same lots or leftovers are often available. Thereby, many lines of inquiries can be followed to identify the suspected food in this setting. Consistent with another study, the use of epidemiological studies was associated with increasing outbreak size [14]. Analytical epidemiological studies are based on comparisons between groups of persons, thus requiring a number of cases that can be studied. The larger the outbreak size, the easier (logistically) an epidemiological study can be conducted and the higher the likelihood of 
Table 2. Characteristics of foodborne disease outbreaks (FBDOs) reported to European Food Safety Authority (EFSA) 2007-2011 associated with evidence from analytical epidemiological study designs, among outbreaks, in which the evidence for the suspected vehicle was considered 'strong'*

\begin{tabular}{|c|c|c|c|}
\hline & OR & $95 \% \mathrm{CI}$ & Significance \\
\hline \multicolumn{4}{|l|}{ Causative agent } \\
\hline Ref.: Salmonella spp. & - & - & - \\
\hline Bacillus spp. & $1 \cdot 72$ & $1 \cdot 14-2 \cdot 58$ & $0 \cdot 01$ \\
\hline Calicivirus & $2 \cdot 36$ & $1 \cdot 64-3 \cdot 40$ & $0 \cdot 00$ \\
\hline Campylobacter spp. & $2 \cdot 70$ & $1 \cdot 69-4 \cdot 32$ & $0 \cdot 00$ \\
\hline Clostridium $\dagger$ & $1 \cdot 10$ & $0.74-1.62$ & $0 \cdot 64$ \\
\hline Histamine & $1 \cdot 51$ & $0 \cdot 99-2 \cdot 31$ & $0 \cdot 06$ \\
\hline Listeria monocytogenes & $1 \cdot 21$ & $0 \cdot 24-6 \cdot 18$ & $0 \cdot 82$ \\
\hline Marine biotoxin & $1 \cdot 55$ & $0 \cdot 72-3 \cdot 33$ & $0 \cdot 27$ \\
\hline Mushroom & $0 \cdot 11$ & $0 \cdot 02-0 \cdot 47$ & $0 \cdot 00$ \\
\hline Other & $1 \cdot 58$ & $0 \cdot 96-2 \cdot 60$ & $0 \cdot 07$ \\
\hline Shigella spp. & $2 \cdot 61$ & $1 \cdot 12-6 \cdot 10$ & 0.03 \\
\hline Staphylococcus & 1.80 & $1 \cdot 33-2 \cdot 44$ & $0 \cdot 00$ \\
\hline Trichinella & $0 \cdot 15$ & $0 \cdot 06-0 \cdot 36$ & 0.00 \\
\hline Unknown & $14 \cdot 88$ & $10 \cdot 30-21 \cdot 50$ & $0 \cdot 00$ \\
\hline \multicolumn{4}{|l|}{ Hospitalizations } \\
\hline Ref.: 0 case & - & - & - \\
\hline 1 case & $0 \cdot 87$ & $0 \cdot 67-1 \cdot 14$ & $0 \cdot 31$ \\
\hline $2-3$ cases & $0 \cdot 85$ & $0 \cdot 66-1 \cdot 10$ & $0 \cdot 22$ \\
\hline$\geqslant 4$ & $0 \cdot 64$ & $0 \cdot 49-0 \cdot 84$ & $0 \cdot 00$ \\
\hline \multicolumn{4}{|l|}{ Outbreak size } \\
\hline Ref.: $0-3$ cases & - & - & - \\
\hline 4-6 cases & 0.95 & $0 \cdot 75-1 \cdot 20$ & $0 \cdot 64$ \\
\hline $7-18$ cases & $1 \cdot 43$ & $1 \cdot 11-1 \cdot 85$ & $0 \cdot 01$ \\
\hline$\geqslant 19$ & $2 \cdot 20$ & $1 \cdot 65-2 \cdot 93$ & 0.00 \\
\hline \multicolumn{4}{|l|}{ Setting } \\
\hline Ref.: Household & - & - & - \\
\hline Commercial food establishment§ & $1 \cdot 42$ & $1 \cdot 13-1 \cdot 78$ & $0 \cdot 00$ \\
\hline Disseminated cases $\|$ & 6.65 & $2 \cdot 91-15 \cdot 21$ & 0.00 \\
\hline Institution & $1 \cdot 61$ & $1 \cdot 23-2 \cdot 11$ & $0 \cdot 00$ \\
\hline Other\# & $1 \cdot 29$ & $0 \cdot 92-1 \cdot 81$ & $0 \cdot 14$ \\
\hline Unknown & 1.28 & $0 \cdot 70-2 \cdot 36$ & $0 \cdot 42$ \\
\hline
\end{tabular}

OR, Odds ratio; CI, confidence interval.

* All ORs adjusted for reporting country and year of notification.

$\dagger$ Clostridium perfringens and Clostridium botulinum.

\$ Household, domestic kitchen, camp, picnic.

$\S$ Restaurant/café/pub/bar/hotel, mobile retailer/market/street vendor, takeaway or fast-food outlet, temporary mass catering (fairs, festivals).

\|Geographically dispersed outbreaks.

\ Canteen or workplace catering, hospital/medical care facilities, residential institution (nursing home, prison, boarding schools), school, kindergarten.

\# Other, farm (primary production) $(n<10)$, aircraft/ship/train $(n<10)$.

distinguishing true effect sizes, thus reducing the likelihood of false-negative results. A minimum numeric threshold for conducting analytical studies cannot be derived from our analysis, because this decision depends on many factors (e.g. severity and dynamic of the outbreak). Notwithstanding, FBDOs in institutions (e.g. restaurant or canteen outbreaks) of a certain size, call for analytical epidemiological studies conducted by local health departments. Simple tools for data entry and analysis, e.g. the linelist tool [8], are available for basic epidemiological comparisons.

In geographically diffuse FBDOs, spanning over more than one jurisdiction, the exact place of exposure is unknown for most or all cases, reducing the possibility of obtaining microbiological evidence. In this situation, analytical epidemiological studies, usually conducted 
by regional or national public health authorities, are often crucial in identifying the suspected food vehicle.

Likewise, if the causative agent is unknown, epidemiological evidence, besides product tracing, is the only means for implicating a particular food vehicle. Similarly, it is difficult to obtain microbiological evidence by end-product testing if the causative agent has a low tenacity (e.g. Campylobacter spp.) or the contaminated food has a short durability (such as fresh produce). This may partially explain why the Campylobacter spp. outbreaks, mainly transmitted by quickly perishable meat or milk products $[15,16]$, were associated with analytical epidemiological evidence. Furthermore, the incubation period of usually 2-5 days [16] prolongs the time span between exposure and outbreak detection, further reducing the chance of finding viable organisms in food products [14].

Our study is subject to a number of limitations. First, we have elucidated FBDO characteristics associated with analytical epidemiological evidence (compared to evidence from microbiological investigations and/or descriptive epidemiology only) by analysing 'successful' investigations, i.e. those that provided strong evidence for a suspected food vehicle. Although we believe the results are plausible, the study design is not suited to infer that we have identified FBDO characteristics where analytical studies should be employed. To this end, FBDO investigations that provided strong evidence for a food vehicle should be compared with those that provided 'weak' or no evidence for a food vehicle. Unfortunately, details of the latter are not routinely collected at the European level. Second, the number of reported investigations of FBDOs in which analytical epidemiological studies have been performed appears unexpectedly high (and have been reported even for outbreaks with $\leqslant 3$ cases). This warrants cautious interpretation of the results. Furthermore, the number varied markedly by country, which necessitated analytical control by reporting country. From 2010 onwards, the unexpectedly high number of investigations of FBDOs with analytical epidemiological evidence for the suspected vehicle decreased substantially (Fig. 1), likely indicating a commendable improvement in the quality of the reported data. Third, the high number of incomplete FBDO reports limits the validity of the results. One variable (place of origin) had to be excluded from analysis due to more than $50 \%$ of missing information.

Analysis of the existing (reported) evidence for the suspected food vehicle elucidates where successful investigations have been conducted in relation to different settings, sizes, causative agents, and other characteristics. This, however, requires careful collection of this information at the local level, surveillance of this information at the national level and, thus far lacking, data quality assurance measures with reference to the foodborne outbreak reporting system at the European level (e.g. automated plausibility checks when uploading data to EFSA). Although epidemiological study designs are indispensable tools in the investigation of FBDOs, barriers exist as they are resource-intense and not suited for every FBDO [8]. Our analysis is the first of its kind and may provide guidance when their use is warranted.

\section{ACKNOWLEDGEMENTS}

The authors thank EFSA for providing the dataset on FBDOs in Europe.

\section{DECLARATION OF INTEREST}

None.

\section{REFERENCES}

1. Tauxe RV, et al. Evolving public health approaches to the global challenge of foodborne infections. International Journal of Food Microbiology 2010; 139 (Suppl. 1): S16-28.

2. Scallan E, et al. Foodborne illness acquired in the United States - major pathogens. Emerging Infectious Diseases 2011; 17: 7-15.

3. Rocourt J, et al. The present state of foodborne disease in OECD countries. Geneva: World Health Organization (WHO); 2003.

4. Lund BM, O'Brien SJ. The occurrence and prevention of foodborne disease in vulnerable people. Foodborne Pathogens and Disease 2011; 8: 961-973.

5. Bouwknegt M, van Pelt W, Havelaar AH. Scoping the impact of changes in population age-structure on the future burden of foodborne disease in the Netherlands, 2020-2060. International Journal of Environmental Research and Public Health 2013; 10: 2888-2896.

6. The European Parliament and the Council of the European Union. Directive 2003/99/EC of the European Parliament and of the Council of 17 November 2003 on the monitoring of zoonoses and zoonotic agents, amending Council Decision 90/424/EEC and repealing Council Directive 92/117/EEC. Official Journal of the European Union 2003; 0000: 31-40.

7. World Health Organization (WHO)- Foodborne Disease Burden Epidemiology Reference Group 20072015. WHO Estimates of the Global Burden of 
Foodborne Diseases. Switzerland: World Health Organization (WHO), 2015.

8. Werber D, Bernard H. Reducing the barriers against analytical epidemiological studies in investigations of local foodborne disease outbreaks in Germany-a starter kit for local health authorities. Euro surveillance 2014; 19: 20714.

9. EFSA (European Food Safety Authority), ECDC (European Centre for Disease Prevention and Control). The European Union summary report on trends and sources of zoonoses, zoonotic agents and food-borne outbreaks in 2012. EFSA Journal 2014; 12: 3547, 3312 pp.

10. Murphree R, et al. Characteristics of foodborne disease outbreak investigations conducted by Foodborne Diseases Active Surveillance Network (FoodNet) sites, 2003-2008. Clinical Infectious Diseases 2012; 54 (Suppl. 5): S498-503.

11. EFSA (European Food Safety Authority). Technical report. Manual for reporting of food-borne outbreaks in accordance with Directive 2003/99/EC from the year 2011. Supporting Publications 2012, EN-265, 249 pp.

12. Palmer S, et al. The role of outbreaks in developing food safety policy: population based surveillance of Salmonella outbreaks in Wales 1986-98. Epidemiology and Infection 2000; 125: 467-472.

13. Palmer SR. Outbreak investigation: the need for 'quick and clean' epidemiology. International Journal of Epidemiology 1995; 24 (Suppl. 1): S34-38.

14. O'Brien SJ, et al. Surveillance of foodborne outbreaks of infectious intestinal disease in England and Wales 1992-1999: contributing to evidence-based food policy? Public Health 2002; 116: 75-80.

15. Forsythe SJ. The Microbiology of Safe Food, 2nd edn. Chichester, UK: Blackwell Publishing, John Wiley and Sons Ltd, 2010.

16. Robert Koch-Institut (RKI). Campylobacter-enteritis. In: RKI-Ratgeber für Ärzte, 2015. 